\title{
REMARKS ON SOME RESULTS OF ALZER, YANG AND TENG
}

\section{JOSIP PEC̆ARIĆ}

H.Alzer [1] proved:

Theorem A. If $u$ and $v$ are non-negative, concave functions defined on $[0,1]$ satisfying

$$
\int_{0}^{1} u^{2 p}(t) d t=\int_{0}^{1} v^{2 q}(t) d t=1, \quad p>0, \quad q>0
$$

then

$$
\int_{0}^{1} u^{p}(t) v^{q}(t) d t \geq \frac{2 \sqrt{(2 p+1)(2 q+1)}}{(p+1)(q+1)}-1 .
$$

G. S. Yang and K. Y. Teng [2] proved:

Theorem B. Let $\alpha>-1 ; p, q>0$. If $u$ and $v$ are nonnegative such that $u\left(t^{\frac{1}{1+\alpha}}\right)$ and $v\left(t^{\frac{1}{1+\alpha}}\right)$ are concave on $[0,1]$ then we have

$$
\begin{aligned}
\int_{0}^{1} u^{p}(t) v^{q}(t) t^{\alpha} d t \geq & {\left[\frac{2}{(p+1)(q+1)}-\frac{1}{\sqrt{(2 p+1)(2 q+1)}}\right](A+1)^{\frac{p}{A}}(B+1)^{\frac{q}{B}} } \\
& \cdot(\alpha+1)^{\frac{p}{A}+\frac{q}{B}-1}\left(\int_{0}^{1} u^{A}(t) t^{\alpha} d t\right)^{\frac{p}{A}}\left(\int_{0}^{1} v^{B}(t) t^{\alpha} d t\right)^{\frac{q}{B}}
\end{aligned}
$$

for $p \leq A \leq 2 p, q \leq B \leq 2 q ;$ and

$$
\begin{aligned}
& \int_{0}^{1} u^{p}(t) v^{q}(t) t^{\alpha} d t \\
\geq & {\left[\frac{2(A+1)^{\frac{p}{A}}(B+1)^{\frac{q}{B}}}{(p+1)(q+1)}-1\right](\alpha+1)^{\frac{p}{A}+\frac{q}{B}-1} \cdot\left(\int_{0}^{1} u^{A}(t) t^{\alpha} d t\right)^{\frac{p}{A}}\left(\int_{0}^{1} v^{B}(t) t^{\alpha} d t\right)^{\frac{q}{B}} }
\end{aligned}
$$

for $A \geq 2 p, B \geq 2 q$.

Moreover, a simple consequence of Theorem 5.2 from [3] is:

Received June 30, 1993. 
Theorem C. Let $p$ and $q$ be nonzero real numbers and let $u$ and $v$ be nonnegative concave functions on $[0,1]$. Let $a \geq 1$ and $b \geq 1$. suppose

(i) $p>0, q>0$; or (ii) $p<0, q<0, p+q>-1, a p>-1, b q>-1$.

Then

$$
\int_{0}^{1} u^{p}(t) v^{q}(t) d t \geq D\left(\int_{0}^{1} u^{p a}(t) d t\right)^{\frac{1}{a}}\left(\int_{0}^{1} v^{q b}(t) d t\right)^{\frac{1}{b}}
$$

where

$$
D=(1+a p)^{1 / a}(1+b q)^{1 / b} B(1+p, 1+q) .
$$

Equality in (4) occurs if

$$
u(t)=t \quad \text { and } \quad v(t)=i-t .
$$

Remark. For $a=b=2$, we have that the best constant in Alzer's inequality (i) is

$$
\sqrt{(2 p+1)(2 q+1)} B(1+p, 1+q) .
$$

Also, the same inequality with the best constant ( 7 ) is also valid if we have: (iii) $-1 / 2<$ $p<0,-1 / 2<q<0$.

Now, we shall prove the following generalization of Theorem B:

Theorem $\mathrm{D}$. Let $\alpha>-1 ; p$ and $q$ be nonzero real numbers and let $u$ and $v$ be nonnegative functions such that $u\left(t^{\frac{1}{(1+\alpha)}}\right)$ and $v\left(t^{\frac{1}{(1+\alpha)}}\right)$ are concave on $[0,1]$. Suppose: (iv) $A \geq p>0, B \geq q>0$; or $(v)-1<A \leq p<0,-1<B \leq q<0$, $p+q>-1$. Then

$$
\int_{0}^{1} u^{p}(t) v^{q}(t) t^{\alpha} d t \geq E\left(\int_{0}^{1} u^{A}(t) t^{\alpha} d t\right)^{\frac{p}{A}}\left(\int_{0}^{1} v^{B}(t) t^{\alpha} d t\right)^{\frac{q}{B}}
$$

where

$$
E=(1+A)^{\frac{p}{A}}(1+B)^{\frac{q}{B}}(\alpha+1)^{\frac{p}{A}+\frac{q}{B}-1} B(1+p, 1+q) .
$$

Equality in (8) occurs if

$$
u(t)=t^{1+\alpha} \quad \text { and } \quad v(t)=1-t^{1+\alpha} .
$$

Proof. We should apply Theorem C on concave functions $u\left(t^{\frac{1}{(1+\alpha)}}\right)$ and $v\left(t^{\frac{1}{(1+\alpha)}}\right)$, and use substitutions: $a p \rightarrow A, q b \rightarrow B$. Then (4) becomes

$$
\int_{0}^{1} u^{p}\left(t^{\frac{1}{1+\alpha}}\right) v^{q}\left(t^{\frac{1}{1+\alpha}}\right) d t \geq D^{\prime}\left(\int_{0}^{1} u^{A}\left(t^{\frac{1}{1+\alpha}}\right) d t\right)^{\frac{p}{A}}\left(\int_{0}^{1} v\left(t^{\frac{1}{1+\alpha}}\right) d t\right)^{\frac{q}{B}}
$$


where $D^{\prime}=(1+A)^{\frac{p}{A}}(1+B)^{\frac{q}{B}} B(1+p, 1+q)$.

Now, substitution $t^{\frac{1}{(1+\alpha)}} \rightarrow t$, gives (8).

It is easy to check that the conditions $a \geq 1, b \geq 1$, (i) and (ii) of Theorem $\mathrm{C}$ give our conditions (iv) and (v).

Remark. Note that in Theorem $B$ in the case of positive $p$ and $q$, the possibility $p \leq A \leq 2 p, B \geq 2 q$ and $q \leq B \leq q, A \geq 2 q$ were not considered. Of course, our constant is the best possible.

\section{References}

[1] H. Alzer, "On an integral inequality of R. Bellman," Tamkang J. Math., 22(1991), 187-191.

[2] G. S. Yang and K. Y. Teng, "A generalization of inverse Schwarz's inequality," Ibid., 23(1992), 117-121.

[3] C. Borell, "Inverse Hölder inequalities in one and several dimensions," J. Math. Anal. Appl., 41(1973), 300-312.

Faculty of Textile-Technology, University of Zagreb, Prilaź Baruna Filipovića 126, 41000 Zagreb, Croatia. 